With I plate

Printed in Great Britain

\title{
The Role of Extracellular Melanoproteins of Venturia inaequalis in Host Susceptibility
}

\author{
By R. C. HIGNETT AND D. S. KIRKHAM \\ East Malling Research Station, East Malling, Maidstone, Kent
}

(Accepted for publication I4 March 1967)

\begin{abstract}
SUMMARY
A series of fungal melanoproteins (mol. wt 10,000-70,000) was isolated from culture filtrates of Venturia inaequalis, and partially characterized by gel filtration and acid hydrolysis. Petiole injection of aqueous solutions of the melanoproteins with non-phytotoxic marker compounds, into apple shoots, produced specific effects on the transport of solutes within the leaves. The effects were not reproduced by substitution of melanoprotein by egg-white lysozyme, rabbit haemoglobin, bovine serum albumen or deoxyribonuclease (EC 3.1.4.5). Inhibition of leaf expansion by injected melanoproteins was observed.

The application of a melanoprotein with spore inoculum of Venturia inaequalis on to leaves of a susceptible apple variety caused a great increase in lesion development. This effect was not reproduced by the use of other proteins. The decreased lesion-stimulating activity of melanoprotein produced by $V$. inaequalis after storage of cultures at $0^{\circ}$ was correlated with decreased effects on solute transport in the host. Radioactive material arising from spore inoculum labelled with $\left[{ }^{4} \mathrm{C}\right] \mathrm{DL}$-alanine was detected in the vascular system of test plants. The distribution of this material in the infected leaf was similar to that given by petiole injection of an indicator compound with melanoprotein in healthy plants.
\end{abstract}

\section{INTRODUCTION}

Work on some obligate fungal parasites of plants has indirectly indicated that during lesion development an initial symbiotic phase occurs in which little or no damage is caused to the host. At an early stage in the formation of visible lesions, before the host tissue becomes necrotic, host metabolites accumulate at the sites of infection (Harvey, 1930; Yarwood \& Jacobson, 1955; Shaw \& Samborski, 1956; Shaw, 1961). It was suggested that products of the infection process contribute to this situation (Shaw \& Samborski, 1956). Venturia inaequalis, being a very specialized yet facultative parasite easily grown in artificial media, was chosen as eminently suitable for studies on the host/pathogen interaction. This paper shows ways in which the pathogen redirects the host metabolism in favour of the developing lesion. Part of this work has already been reported in brief (Hignett \& Kirkham, 1965).

\section{METHODS}

Venturia inaequalis (Cke.) Wint. (clone EI) was grown as described in a previous paper (Kirkham, 1957) single spore isolates on nutrient agar being subcultured on paper cylinders moistened with $10 \%$ (w/v) Oxoid malt extract solution (Oxoid Ltd., 
London). Deep cultures were grown in $500 \mathrm{ml}$. conical flasks containing $300 \mathrm{ml}$. aqueous Oxoid malt extract (Io $\%$, w/v), with continuous magnetic stirring at $16-18^{\circ}$.

Radioactive spores were isolated from paper cylinder cultures grown on a basal medium augmented with $\left[{ }^{14} \mathrm{C}\right] \mathrm{DL}$-alanine at the rate of $0.05 \mathrm{mc} / \mathrm{ml}$. (specific activity $21.7 \mathrm{mc} / \mathrm{m}-\mathrm{mole})$. Loosely bound radioactivity was removed from the spores by thrice repeated washing with distilled water at $18^{\circ}$ in the centrifuge. Labelled spores suspended in $0.5 \mathrm{ml}$. water were applied to the upper surface of leaves of test plants by means of a small paint brush. After incubation for the required period, the spores remaining on the leaf surface were stripped off using a solution of collodion in ether + ethanol $(I+I, v / v)$ which was painted over the whole surface and allowed to dry. By careful peeling of the collodion film, and repetition of the process, over $90 \%$ of the applied spores were removed, as estimated under the microscope.

Greenhouse methods. Test plants were propagated, inoculated and injected through the petioles as previously described (Kirkham \& Hunter, 1965). Inoculation chambers were subdivided with polythene sheet for the application of different treatments.

Autoradiography. Plant material was dried rapidly under high vacuum at room temperature immediately after sampling. Samples were kept flat by application of weighted filter paper. Kodak Kodirex X-ray film was used for autoradiography, being protected from direct contact with the samples by Melinex sheet (gauge 25 type $\mathrm{S}$, I.C.I. Ltd.). Exposure times varied from 7 days to 6 months at room temperature, according to the radioactivity of the sample.

Fungal melanoprotein products. Material was isolated from culture fluids and separated by ethanol fractionation. The fractions insoluble in 50 and $75 \%(\mathrm{v} / \mathrm{v})$ ethanol in water were designated N 50 and N75, respectively. Fraction N 75 was separated on Sephadex G Ioo into four subfractions, labelled I to 4 in order of decreasing molecular weight (range 70,000-10,000; Kirkham \& Hignett, 1966).

Preliminary analysis of the fungal products was done after acid hydrolysis $(6 \mathrm{~N}-$ $\mathrm{HCl}, 107^{\circ}, \mathrm{I} 8 \mathrm{hr}$ ). The black precipitate formed was removed from the mixture, and washed free from acid; it gave a positive reaction to all of the following tests for melanin (Fox \& Kuchnow, 1965).

Colour reactions: (I) bleached by bromine-water, aqueous solutions of $\mathrm{KClO}_{3}+$ $\mathrm{HCl}, \mathrm{KMnO}_{4}$ + oxalic acid, $\mathrm{H}_{2} \mathrm{O}_{2}, \mathrm{CrO}_{3} ;$ (2) dark green colour developed with aqueous $\mathrm{K}_{3} \mathrm{Fe}(\mathrm{CN})_{6} ;$ (3) vapour given off by roasted pigment reddened a pine splinter moistened with concentrated $\mathrm{HCl}$.

Solubility: the black precipitate was soluble in $6 \mathrm{~N}-\mathrm{KOH}$, concentrated $\mathrm{H}_{2} \mathrm{SO}_{4}$, and insoluble in water, $6 \mathrm{~N}-\mathrm{HCl}$ and lipid solvents."

The acid-soluble products of the hydrolysis were dried to remove $\mathrm{HCl}$. Paper chromatography ( $n$-butanol + acetic acid + water $(4+I+I$, by vol.) descending) followed by electrophoresis ( $3 \%$ formic acid $+6 \%$ acetic acid) in the second dimension and development with ninhydrin showed the presence of at least $\mathrm{I} 6$ amino acids. On the basis of this evidence the products were designated as melanoproteins.

Staining procedure. Leaf discs taken at the suspected lesion sites (before sporulation had occurred) and from control regions were digested with pectinase (Light and Co. Ltd.) to isolate the cuticle (Preece, 1962). The presence of subcuticular mycelium was detected by using the Schiff-periodate stain (Preece, 1959).

Transpiration. Differential transpiration rates over infection sites were detected 
by dusting the leaf with anhydrous copper sulphate. Increased transpiration through lesions caused blue patches to appear within $15 \mathrm{~min}$. of application of the anhydrous copper sulphate.

\section{RESULTS}

One-year old potted apple plants (MM. I09 rootstocks), each with a single vigorously growing shoot, were injected with mixed solutions of the melanoproteins and nonphytotoxic indicator compounds ( $\mathrm{I} \%$ acid fuchsin or $\left[{ }^{14} \mathrm{C}\right] \mathrm{D}$-glucose). Two types of distribution pattern within the host leaves were observed. In type I high concentrations of indicator occurred in the vascular system, leaving the interveinal tissue entirely unpermeated. In contrast, control injections without melanoprotein resulted in even permeation of the entire leaf, followed after $24 \mathrm{hr}$ by a decrease in the concentration of indicator in the vascular system. The type II distribution pattern showed fairly even permeation throughout the leaf but with the total uptake being strongly inhibited (see Plate; types I and II, and control).

The effects observed were not reproduced by replacing melanoprotein with bovine serum albumen, egg-white lysozyme, rabbit haemoglobin or deoxyribonuclease (EC 3. I .4 .5). Type I effects were caused by material of fraction $N 75$, also subfractions 3 and 4 , and by a mixture of fractions $\mathrm{N} 75$ and $\mathrm{N} 50$. Type II effects were given by fraction $\mathrm{N} 50$, and by subfractions $I$ and 2 of fraction $\mathrm{N} 75$. A variation of type I effect (type Ia, see P1.) consisted in the accumulation of indicator in the secondary and tertiary veins only. This appearance was produced by the use of melanoprotein at a quarter of the concentration required to give the full type I effect. Type Ia effect was also observed in mature leaves after injection of melanoprotein at a dose rate high enough to give the major vein pattern in young leaves. Slow deterioration of the melanoprotein occurred (with increasing phytoxicity) during storage in aqueous solution at $0^{\circ}$ under toluene.

Use of melanoprotein at concentrations lower than that required to give type Ia effect led to the escape of indicator from the vascular tissues, causing accumulations around the tertiary veins. Injection of melanoprotein at concentrations higher than that required to give the type I effect caused cupping of the leaves, followed by interveinal desiccation and necrosis. Melanoprotein injected at sublethal dose rates partially suppressed leaf growth but not shoot extension. Unevenly permeated leaves grew asymmetrically in the plane of the leaf round the permeated area.

The effects noted above were reproduced in sharply localized areas of the leaf by introducing the melanoprotein and indicator through small wounds. Discrete drops $3 \mathrm{~mm}$. in diameter were applied to the upper surface of leaves between the primary veins, being retained in situ by means of previously applied rings of petroleum jelly. Uptake of liquid by the leaf was effected by piercing the area covered by the drop, with a group of four hypodermic needles mounted on a cork. The effects produced by this technique were restricted to less than one quadrant of the leaf. Pattern types I and II were observed and in addition the type II pattern showed a directional effect in that migration of indicator within the leaf was directed predominantly towards the leaf margin. Indicator applied alone migrated in all directions, and in particular, back to the midrib. The directional effect was investigated in a ro-times replicated experiment on young and on mature leaves. The solutions were applied in each quadrant as closely as possible to the margins of the leaves. In mature leaves only $42 \%$ 
of the controls (treated with indicator only) did not show migration of indicator right back to the midrib, as opposed to $100 \%$ of the test leaves. Migration to the midrib occurred in all young control leaves, but in only $50 \%$ of the young test leaves.

Experiments were done to determine whether metabolite transport in the infected host was altered in ways similar to those described above. For multiple sampling experiments, the second fully expanded leaf (at the time of inoculation) below the shoot tip was designated 0 ; leaves above this being designated $+\mathrm{I},+2$; and $-\mathrm{I},-2$, etc. below. A succession of shoots of Cox's Orange Pippin was injected with $\left[{ }^{14} \mathrm{C}\right] \mathrm{D}-$ glucose ( $5 \mu \mathrm{C}$ total per shoot) at leaves $-2,-3$ and $-4,6$ days after inoculation with Venturia inaequalis, and daily thereafter until lesions appeared. Leaf $+\mathrm{I}$ was sampled

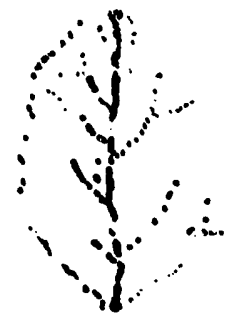

(a)

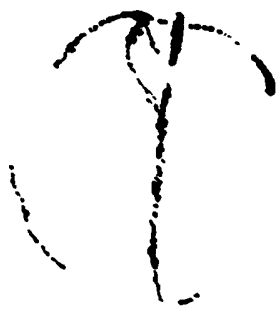

(b)

Fig. I. Diagram of autoradiographs of labelled material injected into host leaf tissue by radioactive spores of Venturia inaequalis. (a) Apple variety MM. I09; upper surface of infected leaf 3 days after inoculation. (b) Apple variety Edward VII; lower surface of infected leaf 12 days after inoculation. Spores were removed in both cases before autoradiography.

$24 \mathrm{hr}$ after injection in each case. The plate (centre and bottom rows) shows a typical selection of autoradiographs from the samples. Type I activity was present at the time of the first sampling (2) and was fully developed 7 days after inoculation. After 12 days, before lesions were visible, $\left[{ }^{14} \mathrm{C}\right] \mathrm{D}$-glucose had preferentially accumulated at the sites of infection (3) (confirmed by staining the cuticle as described under Methods). The final autoradiograph (4) indicates the direct and rapid transport of injected tracer to sporulating lesions, with very little labelling of the rest of the leaf.

The introduction of material into the host by infecting spores was demonstrated by using radioactive spores on shoots of apple varieties MM. I09 and Edward VII. Washed spores taken from cultures grown on $10 \%(w / v)$ malt extract solution augmented with $\left[{ }^{14} \mathrm{C}\right] \mathrm{DL}$-alanine were used as inoculum. Infected leaves were removed and processed as described earlier. Figure $I$ is a diagram of autoradiographs (not reproduced for technical reasons) of the upper surface of an MM. I09 leaf sampled 3 days after inoculation, and of the lower surface of an Edward VII leaf removed 12 days after inoculation, by which time lesions were visible. Both show a type I effect, indicating the introduction of fungal products into the vascular system of the host.

The effect of melanoprotein on lesion development was tested by injecting it into inoculated shoots and by applying it with inoculum. In the former case injections were made $24 \mathrm{hr}$ after inoculation, with a maximum of $1.5 \mathrm{ml}$. (containing up to $15 \mathrm{mg}$. melanoprotein $/ \mathrm{ml}$.) per shoot. In the latter case melanoprotein was added to the inoculum to give final concentrations up to $13 \mathrm{mg}$. $/ \mathrm{ml}$. maximum. 
The results show (Fig. 2) that melanoprotein subfractions 2 and 4 caused when injected into test plants a slight increase in the proportion of leaf area covered by lesions. These results tended to be erratic; stronger lesion stimulation was occasionally observed. In contrast, the same materials when applied with the spore inoculum to the leaf surface showed very consistent and marked lesion-stimulation activity. Culture variability expressed as differences in the patterning activity of the subfraction 4 produced has been noted earlier. Production of less active subfraction 4 was observed, particularly after cultures had been stored under oil for some months. Figure 3 shows the effect on lesion development of adding such material to inoculum used on test plants. Subfraction 4 produced by a freshly re-isolated culture showed markedly higher lesion-stimulation activity as compared to that produced by the same culture after 3 months storage under oil at $0^{\circ}$. The latter product gave results very little different from those of the control experiment. Bovine serum albumen applied with inoculum in a similar way did not influence lesion development. Melanoprotein added (final concentration up to $13 \mathrm{mg} . / \mathrm{ml}$.) to isolated conidia in culture had no effect on germination or growth.

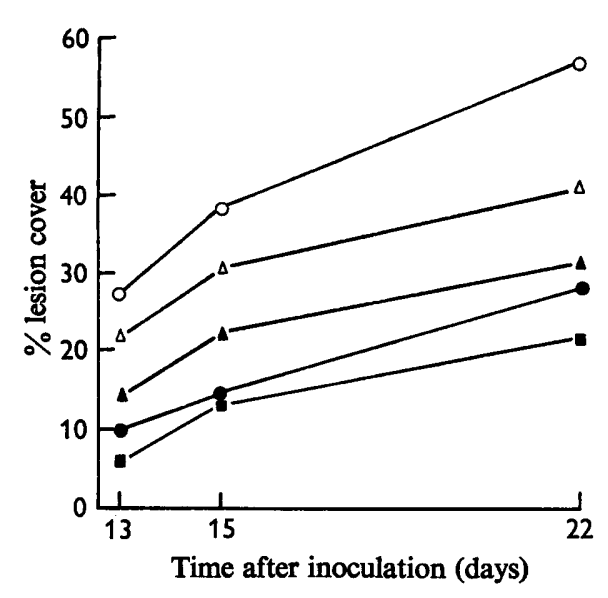

Fig. 2

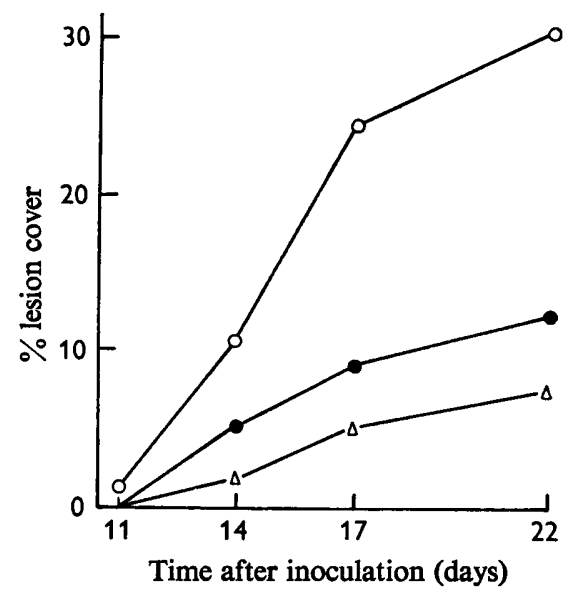

Fig. 3

Fig. 2. Effects of melanoproteins of Venturia inaequalis on lesion development on leaves of apple plants (variety MM. IO9) after inoculation with $V$. inaequalis. O, Subfraction 4 added to inoculum; $\ominus$, subfraction 4 injected $24 \mathrm{hr}$ after inoculation; $\triangle$, subfraction 2 added to inoculum; $\Delta$, subfraction 2 injected $24 \mathrm{hr}$ after inoculation; $\mathbf{\omega}$, control inoculation.

Fig. 3. Comparison of lesion-stimulation activity of melanoprotein produced by old and by freshly re-isolated cultures of Venturia inaequalis. Plants of apple variety MM. 109 were inoculated with spore suspensions containing, respectively, the different melanoprotein subfraction 4 preparations. - , Subfraction 4 produced by cultures after storage; $O$, subfraction 4 produced by re-isolated cultures; $\triangle$, control inoculation. The re-isolated culture was used as a source of spore inoculum in all cases.

\section{DISCUSSION}

The accumulation of metabolites at the site of lesions in parasitized plants has been reported by various authors (Harvey, 1930; Yarwood \& Jacobson, 1955; Shaw \& Samborski, 1956; Shaw, 196I). No evidence was presented to show whether the effect was due either to the host, or to the pathogen. The present work shows that Venturia inaequalis is able to influence host metabolite transport by means of at least two types 
of active extracellular material. The two types of effect on host metabolite transport shown by injected melanoprotein fractions appear to be due to specific properties possessed by them, since other enzymic and non-enzymic proteins tested gave negative results. The Type I activity detected in vivo by using radioactive conidia indicated that this effect was not an artifact of the deep culture method. This experiment also showed that material injected into the host by germinating conidia was not totally catabolized, and persisted in the vascular system and leaf margin up to and including the lesion stage. In contrast, injection of $\left[{ }^{14} \mathrm{C}\right] \mathrm{D}$-glucose at various times after inoculation showed that development of type I activity against host metabolites only occurred during the intermediate stage of lesion development, between infection and sporulation. The vein pattern developed to a maximum intensity before visible lesions appeared, then declined rapidly, giving place to the accumulation of nutrients at infection sites.

The appearance of a vein pattern in rust-infected barley, fed with radioactive compounds before lesion development is apparent in autoradiographs published by Shaw \& Samborski (1956, p. 400, plate VI). As in the present work, a vein pattern developed and declined before visible lesions appeared, giving place to local accumulations of radioactivity. These similarities between the two systems suggest that similar mechanisms may be operating.

The results obtained by directly introducing melanoprotein with indicator compound into the leaf tissue through stab wounds showed that the effects described were not artifacts of the petiole injection method. In addition, the restrictive (type II) activity of melanoprotein fraction $\mathrm{N}_{50}$ had directional properties in that, in contrast to the control experiments, migration of indicator compound towards the midrib was inhibited. The activity of fraction $\mathrm{N}_{50}$ at a given dose-rate was more pronounced in mature leaves; this is to be compared with the greater effect shown by subfraction 4 material (type I activity) on younger leaves. In view of these observations it is suggested that in leaves approaching the lesion stage, i.e. mature leaves, movement of nutrients towards the midrib and out of the leaf is restricted by the action of fraction N 50. This explanation would be consistent with the stunted growth observed during earlier work with leaves permeated with fraction $\mathrm{N}_{50}$ since the normal circulation of metabolites in the host would be restricted (Hignett \& Kirkham, 1965).

The injection of melanoproteins into inoculated plants had little effect on the subsequent development of lesions, although occasional stimulation was observed. However, the onset of necrosis in lesions was often delayed and inhibited. The marked difference in response of the fungus to sprayed application and to petiole injection of subfractions 2 and 4 (Fig. 2) may reflect the critical nature of the dose rate at the site of infection in relation to lesion development. It may also reflect the importance of the direction of travel of the melanoprotein in the vascular system: the directional effect of one melanoprotein fraction has already been mentioned, and penetration by the melanoprotein of the cuticle from the outer surface of the leaf to the site of infection is indicated by the marked lesion stimulation observed. The latter result must be due to interactions at the infection site since fungal products exerted no effects on germination or growth of isolated conidia in culture.

Observation of the more active subfraction 4 produced by freshly re-isolated Venturia inaequalis as compared with that produced from cultures after storage for 3 months under oil at $0^{\circ}$ suggests that the differences might be related to the loss of pathogenicity during storage, which commonly occurs with this organism. 
Journal of General Microbiology, Vol. 48, No. 2

Plate 1

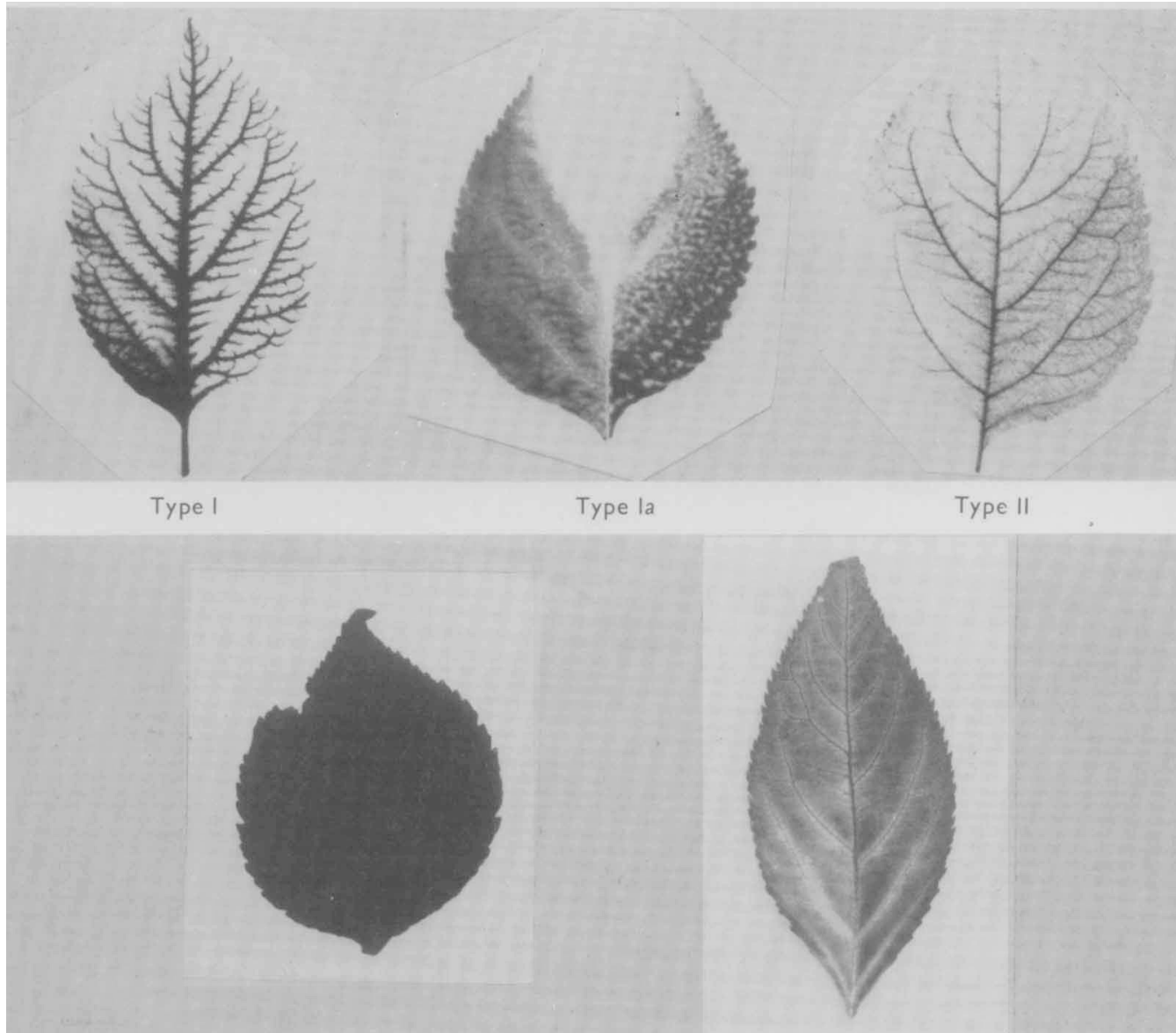

Control

1

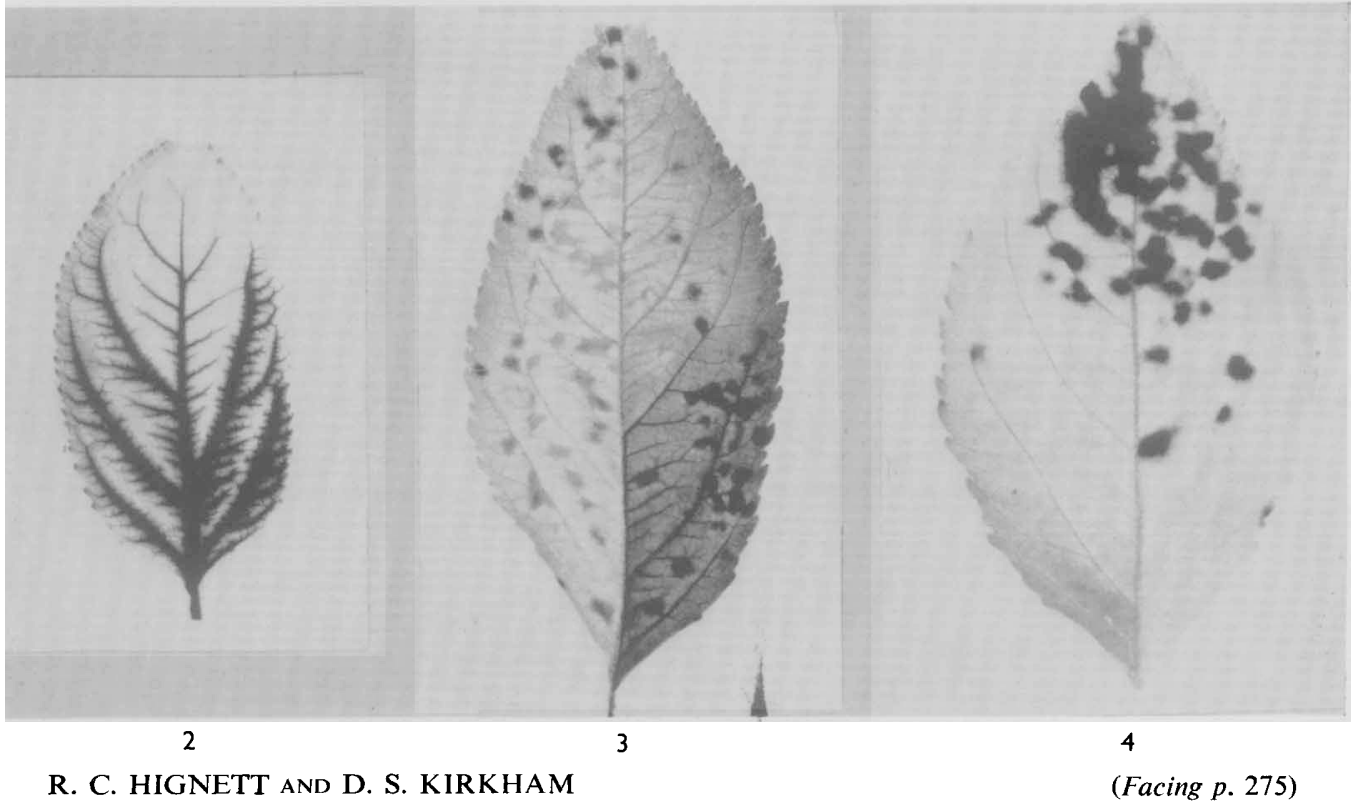


The following mechanism is suggested to account for the observations described. After germination of the conidia and infection of a susceptible host leaf, fungal melanoproteins are produced at the site of infection and spread outwards within the leaf, partially confining metabolites to the vascular system. Thus a specialized transport system of preferred routes is set up leading directly back into the lesion. The export of nutrients from the leaf is progressively restricted, as the leaf matures, by melanoprotein fraction $\mathrm{N}_{5}$. Nutrients are therefore made more available to the developing lesion. Results of the present work suggest that the preferred routes are established long before lesions become visible, and that host metabolites accumulate in them. At the time of lesion eruption, a driving force directed towards the lesion site acts on the accumulated pool of host metabolites in the vascular system. This force is the result of a local increase in transpiration rate as the cuticle above a lesion is lifted and disrupted. Thus the accumulation of nutrients in the vascular system is transferred to the lesion, as indicated by the tracer experiments showing the disappearance of the vein pattern occurring simultaneously with the development of the final lesion pattern.

The technical assistance of Mrs G. F. Sewell, Miss J. Starkey and Mr H. Hutchins is acknowledged.

\section{REFERENCES}

Fox, D. L. \& KuchNow, K. P. (1965). The reversible light-screening pigment of elasmobranch eyes: chemical identity with melanin. Science 150,612 .

HARVEY, R. B. (1930). The relative transpiration rate at infection spots on leaves. Phytopathology 20, 359.

HignetT, R. C. \& KIRKHAM, D. S. (1965). Factors concerned in host specificity of Venturia inaequalis (Cke). Wint. Biochem. J. 97, 34 P.

KIRKHAM, D. S. (1957). Relationships between cultural characters and pathogenicity in Venturia inaequalis and Venturia pirina. J. gen. Microbiol. 16, 360.

KIRKHAM, D. S. \& HignetT, R. C. (1966). Control of host susceptibility and solute transport by metabolites of Venturia inaequalis. Nature, Lond. 2x2, 21 I.

KIRKHAM, D. S. \& HUNTER, L. D. (1965). Studies of the in vivo activity of esters of o-coumaric and cinnamic acids against apple scab. Ann. appl. Biol. 55, 359.

Prezce, T. F. (1959). A staining method for the study of apple scab infections. Plant Pathology 8, 127.

PREECE, T. F. (1962). Removal of apple leaf cuticle by pectinase to reveal the mycelium of Venturia inaequalis (Cke.) Wint. Nature, Lond. 193, 902.

SHAW, M. (1961). The physiology of host-parasite relations. IX. Can. J. Bot. 39, I 393.

Shaw, M. \& SAMBorski, D. J. (1956). The physiology of host-parasite relations I. Can. J. Bot. 34, 389.

YARWOOD, C. E. \& JACOBSON, L. (1955). Accumulation of chemicals in diseased areas of leaves. Phytopathology 45, 43.

\section{EXPLANATION OF PLATE}

Autoradiographed effects of injected melanoproteins of Venturia inaequalis and lesion development, on solute transport in apple leaf tissues.

Top and centre rows. (Apple variety MM. 109). Samples taken $6 \mathrm{hr}$ after injection. Type I. Vein pattern effect produced by injection of indicator substance $\left({ }^{14} \mathrm{C}\right] \mathrm{D}$-glucose) with melanoprotein material N75, or subfractions 3 or 4. Type I a produced by appropriate dilution of injectant used for type I. Type II. Restricted intake of indicator when injected with melanoprotein fraction $\mathrm{N}_{50}$, or subfractions I or 2 isolated from fraction N75. Control. Indicator injected alone.

Centre and bottom rows. (Apple variety Cox's Orange Pippin). Samples taken $24 \mathrm{hr}$ after injection of $\left.{ }^{14} \mathrm{C}\right] \mathrm{D}$-glucose. I. Control. Uninoculated plant. 2. Sample taken 7 days after inoculation with Venturia inaequalis. 3. Sample taken 12 days after inoculation. 4. Sample taken I4 days after inoculation, showng sporulating lesions. 